\title{
BIHARMONIC LAGRANGIAN SUBMANIFOLDS IN KÄHLER MANIFOLDS
}

\author{
SHUN MAETA \\ Division of Mathematics, Graduate School of Information Sciences, \\ Tohoku University, Sendai 980-8579, Japan \\ e-mail:maeta@ims.is.tohoku.ac.jp \\ and HAJIME URAKAWA \\ Institute for International Education, Tohoku University, Sendai 980-8576, Japan \\ e-mail:urakawa@math.is.tohoku.ac.jp
}

(Received 17 March 2012; revised 10 September 2012; accepted 1 November 2012; first published online 25 February 2013)

\begin{abstract}
We give the necessary and sufficient conditions for Lagrangian submanifolds in Kähler manifolds to be biharmonic. We classify biharmonic PNMC Lagrangian $H$-umbilical submanifolds in the complex space forms. Furthermore, we classify biharmonic PNMC Lagrangian surfaces in the two-dimensional complex space forms.
\end{abstract}

\section{Mathematics Subject Classification. Primary 58E20, Secondary 53C43.}

1. Introduction. The theory of harmonic maps has been applied to various fields in differential geometry. Harmonic maps between two Riemannian manifolds are critical points of the energy functional $E(\phi)=\frac{1}{2} \int_{M}\|d \phi\|^{2} v_{g}$ for smooth maps $\phi$ : $M \rightarrow N$. The Euler-Lagrange equation is $\tau(\phi)=0$, where $\tau(\phi)=\operatorname{trace} \nabla d \phi$ is the tension field of $\phi$.

On the contrary, in 1983, Eells and Lemaire [8] proposed the problem to consider polyharmonic maps of order $k$ : they are critical points of the functional

$$
E_{k}(\phi)=\int_{M} e_{k}(\phi) v_{g}, \quad(k=1,2, \ldots),
$$

where $e_{k}(\phi)=\frac{1}{2}\left\|(d+\delta)^{k} \phi\right\|^{2}$ for smooth maps $\phi: M \rightarrow N$, where $\delta$ is the codifferentiation. Jiang [10] studied the first variational formula of the bi-energy $E_{2}$ $(k=2)$, which is written as

$$
E_{2}(\phi)=\int_{M}|\tau(\phi)|^{2} v_{g},
$$

and the critical points of $E_{2}$ are called biharmonic maps. There have been extensive studies on biharmonic maps. Harmonic maps are always biharmonic maps by definition. Here, one of our central problems is to find non-harmonic biharmonic maps. Recently, Sasahara [11] classified the two-dimensional biharmonic Lagrangian submanifolds in the two-dimensional complex space forms.

In this paper, we first show the biharmonic equations for a Lagrangian submanifold $M^{m}$ in a Kähler manifold $\left(N^{m}, J,\langle\cdot, \cdot\rangle\right)$ of complex $m$ dimension (cf. Theorem 3.2). 
We next give the necessary and sufficient conditions for Lagrangian submanifolds in the complex space forms to be biharmonic (cf. Proposition 4.1). In Section 5, we classify biharmonic Lagrangian $H$-umbilical submanifolds in the complex space forms $\left(N^{m}(4 \varepsilon), J,\langle\cdot, \cdot\rangle\right)$ which have the parallel normalized mean curvature vector field (cf. Definition 5.3 and Theorem 5.8). Finally, we classify biharmonic PNMC Lagrangian surfaces in the two-dimensional complex space forms (cf. Theorem 6.2).

2. Preliminaries. In this section, we give necessary notations on biharmonic maps for later use.

Let $\phi:(M, g) \rightarrow(N, h=\langle\cdot, \cdot\rangle)$ be a smooth map from an $m$-dimensional Riemannian manifold $(M, g)$ into an $n$-dimensional Riemannian manifold $(N, h)$. The second fundamental form of $\phi$ is a covariant differentiation $\widetilde{\nabla} d \phi$ of 1 -form $d \phi$, which is a section of $\odot^{2} T^{*} M \otimes \phi^{-1} T N$. For every vector fields $X, Y$ on $M$,

$$
\begin{aligned}
(\tilde{\nabla} d \phi)(X, Y) & =\left(\widetilde{\nabla}_{X} d \phi\right)(Y)=\bar{\nabla}_{X} d \phi(Y)-d \phi\left(\nabla_{X} Y\right) \\
& =\nabla_{d \phi(X)}^{N} d \phi(Y)-d \phi\left(\nabla_{X} Y\right) .
\end{aligned}
$$

Here, $\nabla, \nabla^{N}, \bar{\nabla}$ and $\widetilde{\nabla}$ are the connections on the bundles $T M, T N, \phi^{-1} T N$ and $T^{*} M \otimes \phi^{-1} T N$, respectively.

We consider critical points of the energy functional

$$
E(\phi)=\int_{M} e(\phi) v_{g}
$$

where $e(\phi)=\frac{1}{2}\|d \phi\|^{2}=\frac{1}{2} \sum_{i=1}^{m}\left\langle d \phi\left(e_{i}\right), d \phi\left(e_{i}\right)\right\rangle$ is the energy density of $\phi,\left\{e_{i}\right\}_{i=1}^{m}$ is a locally defined orthonormal frame field on $(M, g)$. Here, $\langle\cdot, \cdot\rangle$ is an induced metric $\phi^{*} h$. The tension field $\tau(\phi)$ of $\phi$ is defined by

$$
\tau(\phi)=\sum_{i=1}^{m}(\widetilde{\nabla} d \phi)\left(e_{i}, e_{i}\right)=\sum_{i=1}^{m}\left(\widetilde{\nabla}_{e_{i}} d \phi\right)\left(e_{i}\right) .
$$

Then, $\phi$ is a harmonic map if and only if $\tau(\phi)=0$.

For the bi-energy $E_{2}$, Jiang [10] showed the first variational formula. $\phi$ is called biharmonic maps if bitension field $\tau_{2}(\phi)$ vanishes, that is,

$$
\tau_{2}(\phi)=\bar{\Delta} \tau(\phi)-\sum_{i=1}^{m} R^{N}\left(\tau(\phi), d \phi\left(e_{i}\right)\right) d \phi\left(e_{i}\right)=0,
$$

where $R^{N}$ is the curvature tensor field, i.e.

$$
R^{N}(U, V) W=\nabla_{U}^{N} \nabla_{V}^{N} W-\nabla_{V}^{N} \nabla_{U}^{N} W-\nabla_{[U, V]}^{N} W, \quad(U, V, W \in \mathfrak{X}(N)),
$$

and $\bar{\Delta}=\bar{\nabla}^{*} \bar{\nabla}=-\sum_{k=1}^{m}\left(\bar{\nabla}_{e_{k}} \bar{\nabla}_{e_{k}}-\bar{\nabla}_{\nabla_{e_{k}} e_{k}}\right)$ is the rough Laplacian.

The Gauss formula is given by

$$
\nabla_{X}^{N} Y=d \phi\left(\nabla_{X} Y\right)+B(X, Y), \quad X, Y \in \mathfrak{X}(M),
$$


where $\mathfrak{X}(M)$ is the space of vector field on $M$, and $B$ denotes the second fundamental form. The Weingarten formula is given by

$$
\nabla_{X}^{N} \xi=-A_{\xi} X+\nabla_{X}^{\perp} \xi, \quad X \in \mathfrak{X}(M), \xi \in \Gamma\left(T M^{\perp}\right),
$$

where $A_{\xi}$ is the shape operator for a normal vector field $\xi$ on $M$ and $\nabla^{\perp}$ denotes the normal connection of the normal bundle on $M$ in $N$. It is well known that the second fundamental form and the shape operator are related by

$$
\langle B(X, Y), \xi\rangle=\left\langle A_{\xi} X, Y\right\rangle .
$$

The equations of Gauss and Codazzi are given by

$$
\begin{gathered}
\left\langle R^{N}(X, Y) Z, W\right\rangle=\langle R(X, Y) Z, W\rangle+\left\langle A_{B(X, Z)} Y, W\right\rangle-\left\langle A_{B(Y, Z)} X, W\right\rangle, \\
\left(\nabla_{X}^{\perp} B\right)(Y, Z)=\left(\nabla_{Y}^{\perp} B\right)(X, Z),
\end{gathered}
$$

where $\nabla^{\perp} B$ is given by

$$
\left(\nabla_{X}^{\perp} B\right)(Y, Z)=\nabla_{X}^{\perp}(B(Y, Z))-B\left(\nabla_{X} Y, Z\right)-B\left(Y, \nabla_{X} Z\right) .
$$

If $\phi:(M, g) \rightarrow(N, h)$ is a biharmonic isometric immersion, then $M$ is called a biharmonic submanifold. In this case, the tension field satisfies that $\tau(\phi)=m \mathbf{H}$, where $\mathbf{H}$ is the harmonic mean curvature vector along $\phi$. The bitension field $\tau_{2}(\phi)$ is rewritten as

$$
\tau_{2}(\phi)=m\left\{\bar{\Delta} \mathbf{H}-\sum_{i=1}^{m} R^{N}\left(\mathbf{H}, d \phi\left(e_{i}\right)\right) d \phi\left(e_{i}\right)\right\},
$$

and $\phi$ is biharmonic if and only if

$$
\bar{\Delta} \mathbf{H}-\sum_{i=1}^{m} R^{N}\left(\mathbf{H}, d \phi\left(e_{i}\right)\right) d \phi\left(e_{i}\right)=0 .
$$

3. The necessary and sufficient conditions for biharmonic Lagrangian submanifolds in Kähler manifolds. In this section, we give the necessary and sufficient conditions for a Lagrangian submanifold in a Kähler manifold to be biharmonic.

Let us recall fundamental materials on a Lagrangian submanifold in a Kähler manifold following Chen and Ogiue [7].

Let $\left(N^{m}, J,\langle\cdot, \cdot\rangle\right)$ be a Kähler manifold of complex dimension $m$, where $J$ is the almost complex structure and $\langle\cdot, \cdot\rangle$ denotes the Kähler metric, which satisfies that $\langle J U, J V\rangle=\langle U, V\rangle$ and $d \Phi=0$, where $\Phi(U, V)=\langle U, J V\rangle, \quad(U, V \in \mathfrak{X}(N))$ is the fundamental 2-form. Let $\left(M^{m}, g\right)$ be a Lagrangian submanifold in a Kähler manifold $\left(N^{m}, J,\langle\cdot, \cdot\rangle\right)$, that is, for all $x \in M, J\left(T_{x} M\right) \subset T_{x} M^{\perp}$, where we also denote that $J$ is the almost complex structure on $M, T_{x} M$ denotes the tangent space of $M$ at $x$ and $T_{x} M^{\perp}$, the normal space at $x$. Then, it is well known that the following three equations hold:

$$
\nabla_{X}^{\perp} J Y=J\left(\nabla_{X} Y\right)
$$




$$
R^{N}(J X, J Y)=R^{N}(X, Y),
$$

for all $X, Y \in \mathfrak{X}(M)$, and

$$
R^{N}(U, V) \cdot J=J \cdot R^{N}(U, V)
$$

for all $U, V \in \mathfrak{X}(N)$.

To show the biharmonic equations for a Lagrangian submanifold in a Kähler manifold, we need the following lemma.

Lemma 3.1. Let $\phi:(M, g) \rightarrow(N,\langle\cdot, \cdot\rangle)$ be an isometric immersion of $(M, g)$ into $(N,\langle\cdot, \cdot\rangle)$. Then, it is biharmonic if and only if

$$
\begin{array}{r}
\operatorname{trace}_{g}\left(\nabla A_{\mathbf{H}}\right)+\operatorname{trace}_{g}\left(A_{\nabla ! \mathbf{H}}(\bullet)\right)-\left(\sum_{i=1}^{m} R^{N}\left(\mathbf{H}, e_{i}\right) e_{i}\right)^{T}=0, \\
\Delta^{\perp} \mathbf{H}+\operatorname{trace}_{g} B\left(A_{\mathbf{H}}(\bullet), \bullet\right)-\left(\sum_{i=1}^{m} R^{N}\left(\mathbf{H}, e_{i}\right) e_{i}\right)^{\perp}=0,
\end{array}
$$

where $(\cdot)^{T}$ is the tangential part and $(\cdot)^{\perp}$ is the normal part.

Proof. Due to (2.3), we have

$$
\bar{\nabla}_{X} \mathbf{H}=-A_{\mathbf{H}}(X)+\nabla_{X}^{\perp} \mathbf{H},
$$

and

$$
\bar{\nabla}_{Y} \bar{\nabla}_{X} \mathbf{H}=-\nabla_{Y} A_{\mathbf{H}}(X)-B\left(Y, A_{\mathbf{H}}(X)\right)+\nabla_{Y}^{\perp} \nabla_{X}^{\perp} \mathbf{H}-A_{\nabla_{\bar{X}}^{\perp} \mathbf{H}} Y,
$$

for all $X, Y \in \mathfrak{X}(M)$.

Thus, we have

$$
\begin{array}{r}
\bar{\Delta} \mathbf{H}=-\sum_{i=1}^{m}\left\{-\nabla_{e_{i}} A_{\mathbf{H}}\left(e_{i}\right)-B\left(e_{i}, A_{\mathbf{H}}\left(e_{i}\right)\right)+\nabla_{e_{i}}^{\perp} \nabla_{e_{i}}^{\perp} \mathbf{H}\right. \\
\left.-A_{\nabla_{e_{i}}^{\perp} \mathbf{H}}\left(e_{i}\right)+A_{\mathbf{H}}\left(\nabla_{e_{i}} e_{i}\right)-\nabla_{\nabla_{e_{i}} e_{i}}^{\perp} \mathbf{H}\right\} .
\end{array}
$$

Dividing this into the tangential and normal part, we obtain Lemma 3.1.

By using Lemma 3.1, we obtain the following theorem.

THEOREM 3.2. Let $\left(N^{m}, J,\langle\cdot, \cdot\rangle\right)$ be a Kähler manifold of complex dimension $m$. Assume that $\phi:\left(M^{m}, g\right) \rightarrow\left(N^{m}, J,\langle\cdot, \cdot\rangle\right)$ is a Lagrangian submanifold. Then, $\phi$ is 
biharmonic if and only if the following two equations hold:

$$
\begin{aligned}
& \operatorname{trace}_{g}\left(\nabla A_{\mathbf{H}}\right)+\operatorname{trace}_{g}\left(A_{\nabla_{\bullet}^{\perp} \mathbf{H}}(\bullet)\right) \\
& -\sum_{i=1}^{m}\left\langle\operatorname{trace}_{g}\left(\nabla_{e_{i}}^{\perp} B\right)-\operatorname{trace}_{g}\left(\nabla_{\bullet}^{\perp} B\right)\left(e_{i}, \bullet\right), \mathbf{H}\right\rangle e_{i}=0, \\
& \Delta^{\perp} \mathbf{H}+\operatorname{trace}_{g} B\left(A_{\mathbf{H}}(\bullet), \bullet\right)+\sum_{i=1}^{m} \operatorname{Ric}^{N}\left(J \mathbf{H}, e_{i}\right) J e_{i} \\
& -\sum_{i=1}^{m} \operatorname{Ric}\left(J \mathbf{H}, e_{i}\right) J e_{i}-J \operatorname{trace}_{g} A_{B(J \mathbf{H}, \bullet)}(\bullet)+m J A_{\mathbf{H}}(J \mathbf{H})=0,
\end{aligned}
$$

where Ric and $\operatorname{Ric}^{N}$ are the Ricci tensor of $\left(M^{m}, g\right)$ and $\left(N^{m},\langle\cdot, \cdot\rangle\right)$ respectively. Here, the trace, trace $g\left(A_{\nabla \bullet \mathbf{H}}(\bullet)\right)$ stand for $\sum_{i=1}^{m} A_{\nabla_{e_{i}}^{\perp} \mathbf{H}}\left(e_{i}\right)$, and so on.

Proof. First note that due to (3.1), the harmonic mean curvature vector $\mathbf{H}$ can be written as $\mathbf{H}=J Z$ for some vector field $Z$ on $M$. By using (3.2) and (3.3), we obtain

$$
\sum_{i=1}^{m}\left\langle R^{N}\left(\mathbf{H}, e_{i}\right) e_{i}, J X\right\rangle=\sum_{i=1}^{m}\left\langle R^{N}\left(Z, J e_{i}\right) J e_{i}, X\right\rangle
$$

which implies that

$$
\sum_{i=1}^{m}\left\langle R^{N}\left(\mathbf{H}, e_{i}\right) e_{i}, J X\right\rangle+\sum_{i=1}^{m}\left\langle R^{N}\left(Z, e_{i}\right) e_{i}, X\right\rangle=\operatorname{Ric}^{N}(Z, X)
$$

By the Gauss equation (2.5), we have

$$
\begin{aligned}
& \sum_{i=1}^{m}\left\langle R^{N}\left(\mathbf{H}, e_{i}\right) e_{i}, J X\right\rangle=\operatorname{Ric}^{N}(Z, X) \\
& -\left\{\sum_{i=1}^{m}\left\langle R\left(Z, e_{i}\right) e_{i}, X\right\rangle+\sum_{i=1}^{m}\left\langle B\left(Z, e_{i}\right), B\left(e_{i}, X\right)\right\rangle-\sum_{i=1}^{m}\left\langle B\left(e_{i}, e_{i}\right), B(Z, X)\right\rangle\right\} \\
= & \operatorname{Ric}^{N}(Z, X)-\operatorname{Ric}(Z, X)-\sum_{i=1}^{m}\left\langle B\left(Z, e_{i}\right), B\left(e_{i}, X\right)\right\rangle+m\langle\mathbf{H}, B(Z, X)\rangle \\
= & -\operatorname{Ric}^{N}(J \mathbf{H}, X)+\operatorname{Ric}(J \mathbf{H}, X) \\
& +\sum_{i=1}^{m}\left\langle B\left(J \mathbf{H}, e_{i}\right), B\left(e_{i}, X\right)\right\rangle-m\langle\mathbf{H}, B(J \mathbf{H}, X)\rangle .
\end{aligned}
$$


From (3.8), we have

$$
\begin{aligned}
& \left(\sum_{i=1}^{m} R^{N}\left(\mathbf{H}, e_{i}\right) e_{i}\right)^{\perp} \\
& =\sum_{j=1}^{m}\left\langle\sum_{i=1}^{m} R^{N}\left(\mathbf{H}, e_{i}\right) e_{i}, J e_{j}\right\rangle J e_{j}=-\sum_{j=1}^{m} \operatorname{Ric}^{N}\left(J \mathbf{H}, e_{j}\right) J e_{j}+\sum_{j=1}^{m} \operatorname{Ric}\left(J \mathbf{H}, e_{j}\right) J e_{j} \\
& \quad+\sum_{i, j=1}^{m}\left\langle B\left(J \mathbf{H}, e_{i}\right), B\left(e_{i}, e_{j}\right)\right\rangle J e_{j}-\sum_{j=1}^{m} m\left\langle\mathbf{H}, B\left(J \mathbf{H}, e_{j}\right)\right\rangle J e_{j}
\end{aligned}
$$

By (2.4), we have

$$
\begin{aligned}
\sum_{i, j=1}^{m}\left\langle B\left(J \mathbf{H}, e_{i}\right), B\left(e_{i}, e_{j}\right)\right\rangle J e_{j} & =\sum_{i, j=1}^{m}\left\langle A_{B\left(J \mathbf{H}, e_{i}\right)}\left(e_{i}\right), e_{j}\right\rangle J e_{j} \\
& =\sum_{i, j=1}^{m}\left\langle J A_{B\left(J \mathbf{H}, e_{i}\right)}\left(e_{i}\right), J e_{j}\right\rangle J e_{j} \\
& =\sum_{i=1}^{m} J A_{B\left(J \mathbf{H}, e_{i}\right)}\left(e_{i}\right) \\
& =J \operatorname{trace}_{g} A_{B(J \mathbf{H}, \bullet}(\bullet),
\end{aligned}
$$

and

$$
\begin{aligned}
\sum_{j=1}^{m}\left\langle\mathbf{H}, B\left(J \mathbf{H}, e_{j}\right)\right\rangle J e_{j} & =\sum_{j=1}^{m}\left\langle A_{\mathbf{H}}(J \mathbf{H}), e_{j}\right\rangle J e_{j} \\
& =\sum_{j=1}^{m}\left\langle J A_{\mathbf{H}}(J \mathbf{H}), J e_{j}\right\rangle J e_{j} \\
& =J A_{\mathbf{H}}(J \mathbf{H}) .
\end{aligned}
$$

Combining (3.9)-(3.11), we obtain

$$
\begin{aligned}
\left(\sum_{i=1}^{m} R^{N}\left(\mathbf{H}, e_{i}\right) e_{i}\right)^{\perp}= & -\sum_{j=1}^{m} \operatorname{Ric}^{N}\left(J \mathbf{H}, e_{j}\right) J e_{j}+\sum_{j=1}^{m} \operatorname{Ric}\left(J \mathbf{H}, e_{j}\right) J e_{j} \\
& +J \operatorname{trace}_{g} A_{B(J \mathbf{H}, \bullet)}(\bullet)-m J A_{\mathbf{H}}(J \mathbf{H}) .
\end{aligned}
$$

By (2.5), we have

$$
\begin{aligned}
\left(\sum_{i=1}^{m} R^{N}\left(\mathbf{H}, e_{i}\right) e_{i}\right)^{T} & =\sum_{i, j=1}^{m}\left\langle R^{N}\left(\mathbf{H}, e_{i}\right) e_{i}, e_{j}\right\rangle e_{j} \\
& =\sum_{i, j=1}^{m}\left\langle\left(\nabla_{e_{j}}^{\perp} B\right)\left(e_{i}, e_{i}\right)-\left(\nabla_{e_{i}}^{\perp} B\right)\left(e_{j}, e_{i}\right), \mathbf{H}\right\rangle e_{j} .
\end{aligned}
$$

Applying Lemma 3.1, we obtain the theorem. 
4. Biharmonic Lagrangian submanifolds in the complex space forms. In this section, we give the necessary and sufficient conditions for Lagrangian submanifolds in the complex space forms to be biharmonic.

Let $N=N^{m}(4 \varepsilon)$ be the simply connected complex $m$-dimensional complex space form of constant holomorphic sectional curvature $4 \varepsilon$. The curvature tensor $R^{N}$ of $N^{m}(4 \varepsilon)$ is given by

$$
\begin{aligned}
& R^{N}(U, V) W \\
& =\varepsilon\{\langle V, W\rangle U-\langle U, W\rangle V+\langle W, J V\rangle J U-\langle W, J U\rangle J V+2\langle U, J V\rangle J W\},
\end{aligned}
$$

for $U, V, W \in \mathfrak{X}(N)$, where $\langle\cdot, \cdot\rangle$ is the Riemannian metric on $N^{m}(4 \varepsilon)$ and $J$ is the almost complex structure of $N^{m}(4 \varepsilon)$. The complex space from $N^{m}(4 \varepsilon)$ is the complex projective space $\mathbb{C} \mathbb{P}^{m}(4 \varepsilon)$, the complex Euclidean space $\mathbb{C}^{m}$ or the complex hyperbolic space $\mathbb{C} \mathbb{H}^{m}(4 \varepsilon)$ according to $\varepsilon>0, \varepsilon=0$ or $\varepsilon<0$.

By using Lemma 3.1, we obtain the following proposition which will be used in the next section.

Proposition 4.1. Let $\left(N^{m}(4 \varepsilon), J,\langle\cdot, \cdot\rangle\right)$ be the complex space form of complex dimension m. Assume that $\phi:\left(M^{m}, g\right) \rightarrow\left(N^{m}(4 \varepsilon), J,\langle\cdot, \cdot\rangle\right)$ is a Lagrangian submanifold. Then, $\phi$ is biharmonic if and only if

$$
\begin{array}{r}
\operatorname{trace}_{g}\left(\nabla A_{\mathbf{H}}\right)+\operatorname{trace}_{g}\left(A_{\nabla_{\bullet}^{\perp} \mathbf{H}}(\bullet)\right)=0, \\
\Delta^{\perp} \mathbf{H}+\operatorname{trace}_{g} B\left(A_{\mathbf{H}}(\bullet), \bullet\right)-(m+3) \varepsilon \mathbf{H}=0 .
\end{array}
$$

Proof. By (4.1), we have

$$
\begin{aligned}
\sum_{i=1}^{m} R^{N}\left(\mathbf{H}, d \phi\left(e_{i}\right)\right) d \phi\left(e_{i}\right)=\varepsilon \sum_{i=1}^{m}\{\langle & \left.d \phi\left(e_{i}\right), d \phi\left(e_{i}\right)\right\rangle \mathbf{H}-\left\langle d \phi\left(e_{i}\right), \mathbf{H}\right\rangle d \phi\left(e_{i}\right) \\
& +\left\langle d \phi\left(e_{i}\right), J d \phi\left(e_{i}\right)\right\rangle J \mathbf{H}-\left\langle d \phi\left(e_{i}\right), J \mathbf{H}\right\rangle J d \phi\left(e_{i}\right) \\
& \left.+2\left\langle\mathbf{H}, J d \phi\left(e_{i}\right)\right\rangle J \phi\left(e_{i}\right)\right\} \\
= & \left\{m \mathbf{H}+\sum_{i=1}^{m}\left\langle\mathbf{H}, J d \phi\left(e_{i}\right)\right\rangle J d \phi\left(e_{i}\right)+2 \mathbf{H}\right\} \\
= & (m+3) \varepsilon \mathbf{H} .
\end{aligned}
$$

By using this and Lemma 3.1, we obtain the proposition.

REMARK 4.2. We remark that Proposition 4.1 is obtained in [9].

5. Biharmonic Lagrangian $H$-umbilical submanifolds in the complex space forms. In this section, we classify biharmonic PNMC (see Definition 5.3) Lagrangian $H$ umbilical submanifolds in the complex space forms. We recall several notions.

Chen introduced the notion of Lagrangian $H$-umbilical submanifolds [5]:

Definition 5.1 [5]. If a Lagrangian submanifold $M$ in a Kähler manifold has the second fundamental form as follows:

$$
\begin{cases}B\left(e_{1}, e_{1}\right)=\lambda J e_{1}, & B\left(e_{i}, e_{i}\right)=\mu J e_{1}, \\ B\left(e_{1}, e_{i}\right)=\mu J e_{i}, & B\left(e_{i}, e_{j}\right)=0, \quad(i \neq j), \quad i, j=2, \ldots, m\end{cases}
$$


for suitable functions $\lambda$ and $\mu$ with respect to some a suitable orthonormal frame field $\left\{e_{1}, \ldots, e_{m}\right\}$ on $M$, then $M$ is called a Lagrangian $H$-umbilical submanifold.

Lagrangian $H$-umbilical submanifolds are the simplest examples of Lagrangian submanifolds next to totally geodesic submanifolds. Since it is known that there are no totally umbilical Lagrangian submanifolds in the complex space forms $N^{m}(4 \varepsilon)$ with $m \geq 2$, we should consider $H$-umbilical Lagrangian submanifolds.

In this case, the harmonic mean curvature vector $\mathbf{H}$ can be denoted by

$$
\mathbf{H}=\frac{\lambda+(m-1) \mu}{m} J e_{1} .
$$

Hereinafter, we put $a=\frac{\lambda+(m-1) \mu}{m}$.

REMARK 5.2. The class of Lagrangian $H$-umbilical submanifolds in the complex space forms includes the following interesting submanifolds:

(1) the Whitney's spheres in the complex Euclidean spaces (cf. [4]),

(2) twistor holomorphic Lagrangian surfaces in the complex projective planes (cf. $[2,5])$.

Furthermore, all Lagrangian $H$-umbilical submanifolds in the complex space forms were classified (cf. [4-6]).

Chen also introduced PNMC submanifolds (cf. [1, 3]):

Definition 5.3 $[\mathbf{1}, \mathbf{3}]$. A submanifold $M$ in a Riemannian manifold is said to have a parallel normalized mean curvature vector field (say, PNMC) if it has nowhere zero mean curvature and the unit vector field in the direction of the mean curvature vector field is parallel in the normal bundle, i.e.

$$
\nabla^{\perp}\left(\frac{\mathbf{H}}{|\mathbf{H}|}\right)=0 .
$$
lemma.

We denote as $\nabla_{e_{i}} e_{j}=\sum_{l=1}^{m} \omega_{j}^{l}\left(e_{i}\right) e_{l}(i, j=1, \ldots, m)$. Then, we obtain the following

LeMmA 5.4 [5, 12]. Let $M^{m}$ be an m-dimensional Lagrangian H-umbilical submanifold in the complex space form. For an orthonormal frame field $\left\{e_{i}\right\}_{i=1}^{m}$, we have

$$
\begin{aligned}
& e_{j} \lambda=(2 \mu-\lambda) \omega_{j}^{1}\left(e_{1}\right), \quad j>1, \\
& e_{1} \mu=(\lambda-2 \mu) \omega_{1}^{l}\left(e_{l}\right), \quad \text { for all } l=2, \ldots m, \\
& (\lambda-2 \mu) \omega_{1}^{i}\left(e_{j}\right)=0, \quad i \neq j>1, \\
& e_{j} \mu=0, \quad j>1, \\
& \mu \omega_{1}^{j}\left(e_{1}\right)=0 \\
& \mu \omega_{1}^{2}\left(e_{2}\right)=\cdots=\mu \omega_{1}^{m}\left(e_{m}\right), \\
& \mu \omega_{1}^{i}\left(e_{j}\right)=0, \quad i \neq j>1 .
\end{aligned}
$$


Proof. By $\left(\nabla_{e_{j}}^{\perp} B\right)\left(e_{1}, e_{1}\right)=\left(\nabla_{e_{1}}^{\perp} B\right)\left(e_{j}, e_{1}\right)$ and (5.1), we obtain (5.3)-(5.5).

By $\left(\nabla_{e_{1}}^{\perp} B\right)\left(e_{j}, e_{j}\right)=\left(\nabla_{e_{j}}^{\perp} B\right)\left(e_{1}, e_{j}\right)$ and (5.1), we obtain (5.6) and (5.7).

By $\left(\nabla_{e_{i}}^{\perp} B\right)\left(e_{j}, e_{j}\right)=\left(\nabla_{e_{j}}^{\perp} B\right)\left(e_{i}, e_{i}\right),(i \neq j>1)$, and (5.1), we obtain (5.8) and (5.9).

By using Lemma 5.4, Sasahara [12] showed the following (see also [13]):

THEOREM 5.5 [12]. Let $\left(N^{m}(4 \varepsilon), J,\langle\cdot, \cdot\rangle\right)$ be the complex space form of complex dimension $m$, where $\varepsilon=\{-1,0,1\}$. Assume that $\phi:\left(M^{m}, g\right) \rightarrow\left(N^{m}(4 \varepsilon), J,\langle\cdot, \cdot\rangle\right)$ is a biharmonic Lagrangian $H$-umbilical submanifold. Then, the mean curvature of $M^{m}$ is non-zero constant if and only if $\varepsilon=1$ and $\phi(M)$ is congruent to an m-dimensional submanifold of $\mathbb{C P}^{m}(4)$ given by

$$
\pi\left(\sqrt{\frac{\mu^{2}}{\mu^{2}+1}} e^{-\frac{i}{\mu} x}, \sqrt{\frac{1}{\mu^{2}+1}} e^{i \mu x} y_{1}, \ldots, \sqrt{\frac{1}{\mu^{2}+1}} e^{i \mu x} y_{m}\right) \subset \mathbb{C P}^{m}(4),
$$

where $x, y_{1}, \ldots, y_{m}$ run through real numbers satisfying $y_{1}^{2}+\cdots+y_{m}^{2}=1$. Here, $\mu=$ $\pm \sqrt{\frac{m+5 \pm \sqrt{m^{2}+6 m+25}}{2 m}}$.

Due to this theorem, we shall classify biharmonic PNMC Lagrangian $H$-umbilical submanifolds in the complex space forms. We shall show the necessary and sufficient conditions for Lagrangian $H$-umbilical submanifolds in the complex space forms to be biharmonic.

Proposition 5.6. Let $\left(M^{m}, g\right)$ be a Lagrangian $H$-umbilical submanifold in the complex space form $\left(N^{m}(4 \varepsilon), J,\langle\cdot, \cdot\rangle\right)$. Then, $M^{m}$ is biharmonic if and only if

$$
\begin{gathered}
2 \lambda\left(e_{1} a\right)+a\left(e_{1} \lambda\right)+\lambda a \sum_{l=2}^{m} \omega_{1}^{l}\left(e_{l}\right)=0, \\
2 \mu\left(e_{j} a\right)+a \lambda \omega_{1}^{j}\left(e_{1}\right)=0, j>1, \\
-\sum_{i=1}^{m} e_{i}\left(e_{i} a\right)+a \sum_{i, j=1}^{m} \omega_{1}^{j}\left(e_{i}\right)^{2}+\sum_{i, j=1}^{m}\left(e_{j} a\right) \omega_{i}^{j}\left(e_{i}\right) \\
+a\left\{\lambda^{2}+(m-1) \mu^{2}-\varepsilon(m+3)\right\}=0 \\
-2 \sum_{i=1}^{m}\left(e_{i} a\right) \omega_{1}^{j}\left(e_{i}\right)-a \sum_{i=1}^{m} e_{i}\left(\omega_{1}^{j}\left(e_{i}\right)\right) \\
-a \sum_{i, l=1}^{m} \omega_{1}^{l}\left(e_{i}\right) \omega_{l}^{j}\left(e_{i}\right)+a \sum_{i, l=1}^{m} \omega_{i}^{l}\left(e_{i}\right) \omega_{1}^{j}\left(e_{l}\right)=0, \quad j>1 .
\end{gathered}
$$


Proof. We shall calculate the tangential part (4.2). By using Lemma 5.4, we have

$$
\begin{aligned}
\operatorname{trace}_{g}\left(A_{\nabla \bullet \mathbf{H}}(\bullet)\right)= & \sum_{i=1}^{m} A_{\nabla_{e_{i}}^{\perp} a J e_{1}} e_{i} \\
= & \sum_{i=1}^{m}\left(e_{i} a\right) A_{J e_{1}} e_{i}+a \sum_{i, l=1}^{m} \omega_{1}^{l}\left(e_{i}\right) A_{J e_{l}} e_{i} \\
= & \lambda \sum_{i=1}^{m}\left(e_{i} a\right) e_{1}+\mu \sum_{i=2}^{m}\left(e_{i} a\right) e_{i} \\
& +a \mu \sum_{l=2}^{m} \omega_{1}^{l}\left(e_{1}\right) e_{l}+a \mu \sum_{l=2}^{m} \omega_{1}^{l}\left(e_{l}\right) e_{1} \\
= & \lambda \sum_{i=1}^{m}\left(e_{i} a\right) e_{1}+\mu \sum_{i=2}^{m}\left(e_{i} a\right) e_{i}+a \mu \sum_{l=2}^{m} \omega_{1}^{l}\left(e_{l}\right) e_{1},
\end{aligned}
$$

and

$$
\begin{aligned}
\operatorname{trace}_{g}\left(\nabla A_{\mathbf{H}}\right)= & \sum_{i=1}^{m} \nabla_{e_{i}}\left(A_{\mathbf{H} e_{i}}\right)-\sum_{i=1}^{m} A_{\mathbf{H}}\left(\nabla_{e_{i}} e_{i}\right) \\
= & \sum_{i=1}^{m} \nabla_{e_{i}}\left(A_{a J e_{1}} e_{i}\right)-a \sum_{i, l=1}^{m} A_{J e_{1}}\left(\omega_{i}^{l}\left(e_{i}\right) e_{l}\right) \\
= & \sum_{i=1}^{m}\left\{\left(e_{i} a\right) A_{J e_{1}} e_{i}+a \nabla_{e_{i}}\left(A_{J e_{1}} e_{i}\right)\right\}-a \sum_{i, l=1}^{m} \omega_{i}^{l}\left(e_{i}\right) A_{J e_{1}} e_{l} \\
= & \lambda\left(e_{1} a\right) e_{1}+a\left(e_{1} \lambda\right) e_{1}+a \lambda \sum_{l=1}^{m} \omega_{1}^{l}\left(e_{1}\right) e_{l} \\
& +\sum_{i=2}^{m}\left\{\mu\left(e_{i} a\right) e_{i}+a\left(e_{i} \mu\right) e_{i}+a \mu \sum_{l=1}^{m} \omega_{i}^{l}\left(e_{i}\right) e_{l}\right\} \\
& -a \lambda \sum_{l=1}^{m} \omega_{l}^{1}\left(e_{l}\right) e_{1}-a \mu \sum_{l=1}^{m} \sum_{i=2}^{m} \omega_{l}^{i}\left(e_{l}\right) e_{i} .
\end{aligned}
$$

By (5.15) and (5.16), we obtain

$$
\begin{aligned}
\operatorname{trace}_{g}\left(\nabla A_{\mathbf{H}}\right)+\operatorname{trace}_{g}\left(A_{\nabla_{\bullet}}(\bullet)\right)= & \left\{2 \lambda\left(e_{1} a\right)+a\left(e_{1} \lambda\right)+a \lambda \sum_{l=2}^{m} \omega_{1}^{l}\left(e_{l}\right)\right\} e_{1} \\
& +\sum_{j=2}^{m}\left\{2 \mu\left(e_{j} a\right)+a \lambda \omega_{1}^{j}\left(e_{1}\right)\right\} e_{j},
\end{aligned}
$$

which yields (5.11) and (5.12). 
We shall calculate the normal part (4.3). By using Lemma 5.4, we have

$$
\begin{aligned}
\Delta^{\perp} \mathbf{H}= & -\sum_{i=1}^{m} \nabla_{e_{i}}^{\perp} \nabla_{e_{i}}^{\perp}\left(a J e_{1}\right)+\sum_{i=1}^{m} \nabla_{\nabla_{e_{i}} e_{i}}^{\perp}\left(a J e_{1}\right) \\
= & -\sum_{i=1}^{m}\left(e_{i} e_{i} a\right) J e_{1}-\sum_{i, j=1}^{m}\left\{2\left(e_{i} a\right) \omega_{1}^{j}\left(e_{i}\right) J e_{j}+a e_{i}\left(\omega_{1}^{j}\left(e_{j}\right) J e_{l}\right)\right\} \\
& -a \sum_{i, j, l=1}^{m} \omega_{1}^{j}\left(e_{i}\right) \omega_{j}^{l}\left(e_{i}\right) J e_{l}+\sum_{i, j=1}^{m} \omega_{1}^{j}\left(e_{i}\right)\left(e_{j} a\right) J e_{1} \\
& +a \sum_{i, j, l=1}^{m} \omega_{i}^{j}\left(e_{i}\right) \omega_{1}^{l}\left(e_{j}\right) J e_{l},
\end{aligned}
$$

and

$$
\operatorname{trace}_{g} B\left(A_{\mathbf{H}}(\bullet), \bullet\right)=a\left\{\lambda^{2}+(m-1) \mu^{2}\right\} J e_{1} .
$$

By (5.18) and (5.19), we obtain

$$
\begin{aligned}
\Delta^{\perp} \mathbf{H} & +\operatorname{trace}_{g} B\left(A_{\mathbf{H}}(\bullet), \bullet\right)-(m+3) \varepsilon \mathbf{H} \\
= & \left\{-\sum_{i=1}^{m} e_{i} e_{i} a+a \sum_{i, j=1}^{m} \omega_{1}^{j}\left(e_{i}\right)^{2}+\sum_{i, j=1}^{m}\left(e_{j} a\right) \omega_{i}^{j}\left(e_{i}\right)\right. \\
& \left.+a\left\{\lambda^{2}+(m-1) \mu^{2}-\varepsilon(m+3)\right\}\right\} J e_{1} \\
& +\sum_{j=2}^{m}\left\{-2 \sum_{i=1}^{m}\left(e_{i} a\right) \omega_{1}^{j}\left(e_{i}\right)-a \sum_{i=1}^{m} e_{i}\left(\omega_{1}^{j}\left(e_{i}\right)\right)\right. \\
& \left.-a \sum_{i, l=1}^{m} \omega_{1}^{l}\left(e_{i}\right) \omega_{l}^{j}\left(e_{i}\right)+a \sum_{i, l=1}^{m} \omega_{i}^{l}\left(e_{i}\right) \omega_{1}^{j}\left(e_{l}\right)\right\} J e_{j},
\end{aligned}
$$

which yields (5.13) and (5.14).

From Proposition 5.6, we obtain the following proposition.

Proposition 5.7. Let $\left(M^{m}, g\right)$ be a Lagrangian H-umbilical submanifold in the complex space form $\left(N^{m}(4 \varepsilon), J,\langle\cdot, \cdot\rangle\right)$. Then, $M^{m}$ is (non-harmonic) biharmonic if and only if $\mu \neq 0$ and

$$
\begin{gathered}
2 \lambda\left(e_{1} a\right)+a\left(e_{1} \lambda\right)+a \lambda(m-1) k=0 \\
e_{j} a=0, \quad j>1, \\
-e_{1}\left(e_{1} a\right)+a(m-1) k^{2}-\left(e_{1} a\right)(m-1) k+a\left\{\lambda^{2}+(m-1) \mu^{2}-\varepsilon(m+3)\right\}=0, \\
e_{j} k=0, \quad j>1,
\end{gathered}
$$


where, $k=\omega_{1}^{2}\left(e_{2}\right)=\cdots=\omega_{1}^{m}\left(e_{m}\right)$.

Proof. We shall show $\mu \neq 0$. If $\mu=0$, then $a=\frac{1}{m} \lambda \neq 0$. By Lemma 5.4, we have

$$
\omega_{1}^{i}\left(e_{j}\right)=0, j=2, \ldots, m .
$$

From (5.11), $e_{1} a=0$. From (5.12), we obtain

$$
\omega_{1}^{j}\left(e_{1}\right)=0, j=1, \ldots, m .
$$

Combining (5.24) and (5.25), we have

$$
\omega_{1}^{i}\left(e_{j}\right)=0, \quad i, j=1, \ldots, m .
$$

It follows that $\left\langle R\left(e_{1}, e_{i}\right) e_{i}, e_{1}\right\rangle=0$. Thus, by (2.5), we have $\varepsilon=0$. By (5.3), we have $e_{j} a=$ $0,(j>1)$. From these and (5.13), we obtain $a=0$, which contradicts the assumption.

We only have to consider the case of $\mu \neq 0$. Then, we have

$$
\begin{array}{r}
\omega_{1}^{i}\left(e_{j}\right)=0, \quad i \neq j, \\
\omega_{1}^{2}\left(e_{2}\right)=\cdots=\omega_{1}^{m}\left(e_{m}\right) .
\end{array}
$$

We put $k=\omega_{1}^{2}\left(e_{2}\right)=\cdots=\omega_{1}^{m}\left(e_{m}\right)$.

By (5.28), we can denote that the equation (5.11) is (5.20). Putting (5.27) into (5.12), we obtain (5.21). From (5.21) and (5.13), we have (5.22). Putting (5.21) into (5.14), we have

$$
-a \sum_{i=1}^{m} e_{i}\left(\omega_{1}^{j}\left(e_{i}\right)\right)-a \sum_{i, l=1}^{m} \omega_{1}^{l}\left(e_{i}\right) \omega_{l}^{j}\left(e_{i}\right)+a \sum_{i, l=1}^{m} \omega_{i}^{l}\left(e_{i}\right) \omega_{1}^{j}\left(e_{l}\right)=0 .
$$

Form this and (5.27), we obtain (5.23).

By using Theorem 5.5, we shall classify all the biharmonic PNMC Lagrangian $H$-umbilical submanifolds in the complex space forms.

THEOREM 5.8. Let $\left(N^{m}(4 \varepsilon), J,\langle\cdot, \cdot\rangle\right)$ be the complex space form of complex dimension $m$, where $\varepsilon=\{-1,0,1\}$. Assume that $\phi:\left(M^{m}, g\right) \rightarrow\left(N^{m}(4 \varepsilon), J,\langle\cdot, \cdot\rangle\right)$ is a Lagrangian $H$-umbilical submanifold which has PNMC. Then, $\phi$ is biharmonic if and only if $\varepsilon=1$ and $\phi(M)$ is congruent to an m-dimensional submanifold of $\mathbb{C P} \mathbb{P}^{m}(4)$ given by

$$
\pi\left(\sqrt{\frac{\mu^{2}}{\mu^{2}+1}} e^{-\frac{i}{\mu} x}, \sqrt{\frac{1}{\mu^{2}+1}} e^{i \mu x} y_{1}, \ldots, \sqrt{\frac{1}{\mu^{2}+1}} e^{i \mu x} y_{m}\right) \subset \mathbb{C P}^{m}(4),
$$

where $x, y_{1}, \ldots, y_{m}$ run through real numbers satisfying $y_{1}{ }^{2}+\cdots+y_{m}{ }^{2}=1$. Here, $\mu=$ $\pm \sqrt{\frac{m+5 \pm \sqrt{m^{2}+6 m+25}}{2 m}}$.

REMARK 5.9. The biharmonic immersion in $\mathbb{C P}^{m}$, given in (5.29), has a parallel mean curvature vector field, i.e. $\nabla^{\perp} \mathbf{H}=0$.

Proof. By the assumption

$$
\nabla^{\perp}\left(\frac{\mathbf{H}}{|\mathbf{H}|}\right)=\nabla^{\perp}\left(\frac{a J e_{1}}{|a|}\right)=0,
$$


and $a \neq 0$, we have

$$
J\left(\nabla e_{1}\right)=\nabla^{\perp} J e_{1}=0 .
$$

Thus, we obtain

$$
0=\nabla_{e_{i}} e_{1}=\sum_{l=1}^{m} \omega_{1}^{l}\left(e_{i}\right) e_{l}, \quad(i=1, \ldots, m),
$$

which implies that

$$
\omega_{1}^{l}\left(e_{i}\right)=0, \quad(i, l=1, \ldots, m)
$$

In particular, we have

$$
k=\omega_{1}^{2}\left(e_{2}\right)=\cdots=\omega_{1}^{m}\left(e_{m}\right)=0 .
$$

By (5.4), we obtain

$$
e_{1} \mu=0
$$

Combining this and (5.6), $\mu$ is a constant. Since $\left\langle R\left(e_{i}, e_{1}\right) e_{1}, e_{i}\right\rangle=0$, we have

$$
\mu^{2}-\lambda \mu=\varepsilon
$$

Thus, $\lambda$ is a constant. Therefore, $a=\frac{\lambda+(m-1) \mu}{m}$ is a non-zero constant.

By using Theorem 5.5, we obtain (5.29).

Conversely, by a direct computation, it turns out that the immersion (5.29) is a biharmonic PNMC Lagrangian immersion.

REMARK 5.10. (1) We cannot answer whether or not the same conclusion of Theorem 5.8 holds without the assumption PNMC.

(2) If $\mu_{+}=\mu_{0}$, and $\mu_{-}=-\mu_{0}$, where $\mu_{0}=\sqrt{\frac{m+5 \pm \sqrt{m^{2}+6 m+25}}{2 m}}$, then it seems that the corresponding submanifolds to $\mu_{+}$and $\mu_{-}$are isometric each other.

6. Biharmonic PNMC surface. In this section, we classify all the biharmonic PNMC Lagrangian surfaces in the two-dimensional complex space forms $\left(N^{2}(4 \varepsilon), J,\langle\cdot, \cdot\rangle\right)$.

Let $\phi: M^{2} \rightarrow\left(N^{2}(4 \varepsilon), J,\langle\cdot, \cdot\rangle\right)$ be a Lagrangian surface. Let $\left\{e_{1}, e_{2}\right\}$ be an orthonormal frame field on $M^{2}$ such that $J e_{1}$ is parallel to $\mathbf{H}$. Then, the second fundamental form is

$$
\begin{aligned}
& B\left(e_{1}, e_{1}\right)=(a-b) J e_{1}+c J e_{2}, \\
& B\left(e_{1}, e_{2}\right)=c J e_{1}+b J e_{2}, \\
& B\left(e_{2}, e_{2}\right)=b J e_{1}-c J e_{2},
\end{aligned}
$$


for some functions $a(\neq 0), b$ and $c$. We put $\nabla_{e_{1}} e_{1}=\alpha e_{2}$ and $\nabla_{e_{2}} e_{1}=\beta e_{2}$ (then, we have $\nabla_{e_{1}} e_{2}=-\alpha e_{1}$ and $\left.\nabla_{e_{2}} e_{2}=-\beta e_{1}\right)$. From these, we have

$$
\begin{aligned}
& \left(\nabla_{e_{1}}^{\perp} B\right)\left(e_{2}, e_{2}\right)=\left(e_{1} b+3 c \alpha\right) J e_{1}-\left(e_{1} c-3 b \alpha\right) J e_{2}, \\
& \left(\nabla_{e_{2}}^{\perp} B\right)\left(e_{1}, e_{2}\right)=\left\{e_{2} c+(a-3 b) \beta\right\} J e_{1}+\left(e_{2} b+3 c \beta\right) J e_{2}, \\
& \left(\nabla_{e_{1}}^{\perp} B\right)\left(e_{1}, e_{2}\right)=\left\{e_{1} c+(a-3 b) \alpha\right\} J e_{1}+\left(e_{1} b+3 c \alpha\right) J e_{2}, \\
& \left(\nabla_{e_{2}}^{\perp} B\right)\left(e_{1}, e_{1}\right)=\left\{e_{2}(a-b)-3 c \beta\right\} J e_{1}+\left\{e_{2} c+(a-3 b) \beta\right\} J e_{2} .
\end{aligned}
$$

By using (2.6), we obtain

$$
\begin{aligned}
e_{1} b+3 c \alpha & =e_{2} c+(a-3 b) \beta, \\
-e_{1} c+3 b \alpha & =e_{2} b+3 c \beta, \\
e_{2}(a-b)-3 c \beta & =e_{1} c+(a-3 b) \alpha .
\end{aligned}
$$

Combining (6.3) and (6.4) leads to

$$
e_{2} a=a \alpha
$$

From (2.5), we have

$$
a b-2 b^{2}-2 c^{2}+\varepsilon=-\alpha^{2}-\beta^{2}+e_{2} \alpha-e_{1} \beta .
$$

By using these results, we obtain the following proposition.

Proposition 6.1. Let $\left(N^{2}(4 \varepsilon), J,\langle\cdot, \cdot\rangle\right)$ be the two-dimensional complex space form. Assume that $\phi: M^{2} \rightarrow\left(N^{2}(4 \varepsilon), J,\langle\cdot, \cdot\rangle\right)$ is a Lagrangian surface. Then $\phi$ is biharmonic if and only if

$$
\begin{aligned}
& 3\left(e_{1} a\right) a-2\left(e_{1} a\right) b+4 a c \alpha+2 a b \beta=0, \\
& 2\left(e_{1} a\right) c+4 a \alpha b+a^{2} \alpha-2 a c \beta=0, \\
& -e_{1} e_{1} a-\beta\left(e_{1} a\right)+a\left(-5 \epsilon+(a-b)^{2}+b^{2}+2 c^{2}+\alpha^{2}+\beta^{2}-e_{2} \alpha\right)=0, \\
& 2\left(e_{1} a\right) \alpha+a\left(2 \alpha \beta+e_{1} \alpha+e_{2} \beta-a c\right)=0 .
\end{aligned}
$$

Proof. We shall calculate the tangential part (4.2). Since $A_{J e_{1}} e_{1}=(a-b) e_{1}+c e_{2}$, $A_{J e_{1}} e_{2}=A_{J e_{2}} e_{1}=c e_{1}+b e_{2}$ and $A_{J e_{2}} e_{2}=b e_{1}-c e_{2}$, we have

$$
\begin{aligned}
\operatorname{trace}_{g}\left(A_{\nabla ! \mathbf{H}}(\bullet)\right)= & \sum_{i=1}^{2} A_{\nabla_{e_{i}}^{\perp} \mathbf{H}}\left(e_{i}\right) \\
= & \frac{1}{2}\left\{\left(e_{1} a\right)(a-b)+\left(e_{2} a\right) c+a \alpha c+a \beta b\right\} e_{1} \\
& +\frac{1}{2}\left\{\left(e_{1} a\right) c+\left(e_{2} a\right) b+a \alpha b-a \beta c\right\} e_{2},
\end{aligned}
$$


and

$$
\begin{aligned}
& \operatorname{trace}_{g}\left(\nabla A_{\mathbf{H}}\right)=\sum_{i=1}^{2}\left(\nabla_{e_{i}} A_{\mathbf{H}}\right) e_{i}=\sum_{i=1}^{2}\left\{\nabla_{e_{i}}\left(A_{\mathbf{H}} e_{i}\right)-A_{\mathbf{H}}\left(\nabla_{e_{i}} e_{i}\right)\right\} \\
& =\frac{1}{2}\left\{\left(e_{1} a\right)(a-b)+a\left(e_{1} a\right)-a\left(e_{1} b\right)-a \alpha c\right. \\
& \left.\quad+\left(e_{2} a\right) c+a\left(e_{2} c\right)-a b \beta-a \alpha c+a \beta(a-b)\right\} e_{1} \\
& +\frac{1}{2}\left\{\left(e_{1} a\right) c+a(a-b) \alpha+a\left(e_{1} c\right)+\left(e_{2} a\right) b+a\left(e_{2} b\right)+a c \beta-a \alpha b+a \beta c\right\} e_{2} .
\end{aligned}
$$

By using (6.2)-(6.5), we obtain (6.7) and (6.8).

We shall calculate the normal part (4.3). We have

$$
\begin{aligned}
\Delta^{\perp} \mathbf{H}= & -\frac{1}{2}\left\{e_{1}\left(e_{1} a\right) J e_{1}+2\left(e_{1} a\right) \alpha J e_{2}+a\left(e_{1} \alpha\right) J e_{2}-a \alpha^{2} J e_{1}-\alpha\left(e_{2} a\right) J e_{1}\right\} \\
& -\frac{1}{2}\left\{e_{2}\left(e_{2} a\right) J e_{1}+2\left(e_{2} a\right) \beta J e_{2}+a\left(e_{2} \beta\right) J e_{2}-a \beta^{2} J e_{1}+\beta\left(e_{1} a\right) J e_{1}\right\},
\end{aligned}
$$

and

$$
\sum_{i=1}^{2} B\left(A_{\mathbf{H}} e_{i}, e_{i}\right)=\frac{1}{2} a\left\{(a-b)^{2} J e_{1}+(a-b) c J e_{2}+2 c^{2} J e_{1}+b c J e_{2}+b^{2} J e_{1}\right\} .
$$

By using (6.5), we obtain (6.9) and (6.10).

We shall classify all the biharmonic PNMC Lagrangian surfaces in the twodimensional complex space forms.

THEOREM 6.2. Let $\left(N^{2}(4 \varepsilon), J,\langle\cdot, \cdot\rangle\right)$ be the two-dimensional complex space form. Assume that $\phi: M^{2} \rightarrow\left(N^{2}(4 \varepsilon), J,\langle\cdot, \cdot\rangle\right)$ is a biharmonic Lagrangian surface. Then, the following properties are equivalent:

(1) the mean curvature is a non-zero constant;

(2) $M^{2}$ has PNMC.

Moreover, if the biharmonic Lagrangian surface has the condition (1) or (2), then we have $\epsilon>0$ and $M^{2}$ is a $H$-umbilical surface. If $\varepsilon=1, \phi$ is locally given by

$$
\begin{aligned}
& \phi(x, y)= \\
& \pi\left(\sqrt{\frac{b^{2}}{b^{2}+1}} \mathrm{e}^{-\frac{i}{b} x}, \sqrt{\frac{1}{b^{2}+1}} \mathrm{e}^{i b x} \cos \sqrt{b^{2}+1} y, \sqrt{\frac{1}{b^{2}+1}} \mathrm{e}^{i b x} \sin \sqrt{b^{2}+1} y\right),
\end{aligned}
$$

where $b= \pm \frac{\sqrt{7 \pm \sqrt{41}}}{2}$.

Proof. We shall show (1) $\Rightarrow(2)$. Since the mean curvature $a$ is a non-zero constant, from (6.5), we have $\alpha=0$. From these, we have that (6.7) is $b \beta=0$, and (6.8) is $c \beta=0$. If $\beta \neq 0$, then we have $b=c=0$. But, from (6.2), we have $\beta=0$. This contradicts our assumption $\beta \neq 0$. Therefore, we obtain $\beta=0$, which means that $M^{2}$ has PNMC.

We shall show that (2) $\Rightarrow(1)$. By the assumption

$$
\nabla^{\perp}\left(\frac{\mathbf{H}}{|\mathbf{H}|}\right)=\nabla^{\perp}\left(\frac{a J e_{1}}{|a|}\right)=0,
$$


and $a \neq 0$, we have

$$
J\left(\nabla e_{1}\right)=\nabla^{\perp} J e_{1}=0 .
$$

Thus, we obtain

$$
0=\nabla_{e_{i}} e_{1}= \begin{cases}\alpha e_{2} & (i=1), \\ \beta e_{2} & (i=2),\end{cases}
$$

which implies that $\alpha=0$ and $\beta=0$. From (6.7), we have that $e_{1} a=0$ or $3 a-2 b=0$.

The case of $e_{1} a=0$. By (6.5), $a$ is constant.

The case of $3 a-2 b=0$. From (6.10), we have $c=0$. It follows from $c=0$, (6.2) and (6.3) that $b$ is constant. By combining (6.6) and (6.9), we have $a=0$. This contradicts our assumption $a \neq 0$.

Therefore $a$ is constant.

If $M^{2}$ has the condition (1) or (2), from (6.10), we have $c=0$, i.e., $M^{2}$ is a $H$-umbilical surface. By using Theorem 5.8, we obtain the theorem.

ACKnowledgements. We thank Professor Kazuo Akutagawa for many helpful advice and Professor Toru Sasahara for crucial comments to the first draft. We also thank the referee for useful comments. S.M. also thanks Professor Ye-Lin Ou for useful comments. S.M. is supported by Research Fellowships of the Japan Society for the Promotion of Science for Young Scientists (No. 23-6949). H.U. is supported by the Grants-in-Aid for Scientific Research (C), Japan Society for the Promotion of Science (No. 21540207).

\section{REFERENCES}

1. A. Balmus, S. Montaldo and C. Oniciuc, Biharmonic PNMC submanifolds in spheres, to appear in Ark. Mat.

2. I. Castro and F. Urbano, Twistor holomorphic Lagrangian surfaces in complex projective and hyperbolic planes, Ann. Global. Anal. Geom. 13 (1995), 59-67.

3. B. Y. Chen, Surfaces with parallel normalized mean curvature vector, Mon. Math. 90 (1980), 185-194.

4. B. Y. Chen, Complex extensors and Lagrangian submanifolds in complex Euclidean spaces, Tohoku Math. J. 49 (1997), 277-297.

5. B. Y. Chen, Interaction of Legendre curves and Lagrangian submanifolds, Israel $J$. Math. 99 (1997), 69-108.

6. B. Y. Chen, Representation of flat Lagrangian $H$-umbilical submanifolds in complex Euclidean spaces, Tohoku Math. J. 51 (1999), 13-21.

7. B. Y. Chen and K. Ogiue, On totally real submanifolds, Trans. Amer. Math. Soc. 193 (1974), 257-266.

8. J. Eells and L. Lemaire, Selected topics in harmonic maps, CBMS Reg. Conf. Ser., vol. 50 (AMS, Providence, RI, 1983).

9. D. Fetcu, E. Loubeau, S. Montaldo and C. Oniciuc, Biharmonic submanifolds of $\mathbb{C} P^{n}$, Math. Z. 266(3) (2010), 505-531.

10. G. Y. Jiang, 2-harmonic maps and their first and second variational formulas, Chinese Ann. Math. A 7 (1986), 388-402 [English translation, Note Mat. 28 (2009), 209-232].

11. T. Sasahara, Biharmonic Lagrangian surfaces of constant mean curvature in complex space forms, Glasgow Math. J. 49 (2007), 497-507.

12. T. Sasahara, Biminimal Lagrangian $H$-umbilical submanifolds in complex space forms, Geom. Dedicata 160 (2012), 185-193.

13. T. Sasahara, A classification result for biminimal Lagrangian surfaces in complex space forms, J. Geom. Phys. 60 (2010), 884-895. 